\title{
Unified data Embedding and Scrambling Advancement in Reversible Watermarking
}

\author{
P S Malage ${ }^{1}$, A M Bedarkar ${ }^{2}$ \\ Asst. Professor, Dept of Electronics Engineering, Walchand Institute of Technology, Solapur, India ${ }^{1}$ \\ PG Scholar, Dept of Electronics Engineering, Walchand Institute of Technology, Solapur, India ${ }^{2}$
}

\begin{abstract}
In general, a data hiding technique for digital content can be classified into data embedding and perceptual encryption (Scrambling). Data embedding techniques focuses on high-output image quality so that the difference between the original and the embedded images is imperceptible to the naked eye. As a new trend some researcher explores the reversible data embedding technique to get higher payload on the cost of degradation in perceptual quality of the output image. This paper proposed a unique technique i.e Unified data embedding-scrambling technique called UES to achieve two objectives simultaneously, namely, high payload and adaptive scalable quality degradation. First Checkerboard based prediction method is proposed for pixel intensity value prediction. In this method $75 \%$ of the pixel is predicted with reference to information obtained from $25 \%$ of the image. Then based upon prediction error calculated, locations of predicted pixels are vacated to embed information. In addition, the prediction errors are stored at a predetermined precision using the structure side information to perfectly reconstruct or approximate the original image. The precision of the stored prediction errors can be adjusted to control the perceptual quality of the reconstructed image. Experimental results confirm that the proposed UES algorithm is able to reconstruct the original image after completely degrading its perceptual quality.
\end{abstract}

Keywords: Data hiding, Embedding, Scrambling, Precision error.

\section{INTRODUCTION}

In recent years, the Internet has become a popular public communication channel to transmit large amounts of multimedia information, such as text, images, and audio. However, the prevalence of the Internet has caused some security concerns, including protecting private communication and intellectual property rights (IPR) and hiding needs. Media can avoid the hacker's notice and prevent a possible attack. Cryptography is a well-known method for solving such security problems [1,2], but, since the encrypted secret data generated by this kind of method is unreadable, it may attract the attention of eavesdroppers. In order to solve this problem, the information hiding method is proposed that can utilize a variety of multimedia as cover media and embed the secret data into the cover media to generate stego media. Because of the visual similarity of stego media and cover media, the stego can avoid the hacker's notice and prevent a possible attack. In general, while considering a data hiding technique for digital content conventional data embedding aims to utilize content (e.g., image) as a venue to host external information maintaining the perceptual quality of the image. But the purpose of other approach i.e perceptual encryption (Scrambling) is to make content imperceptible by converting it into a severely distorted or meaningless form [3].

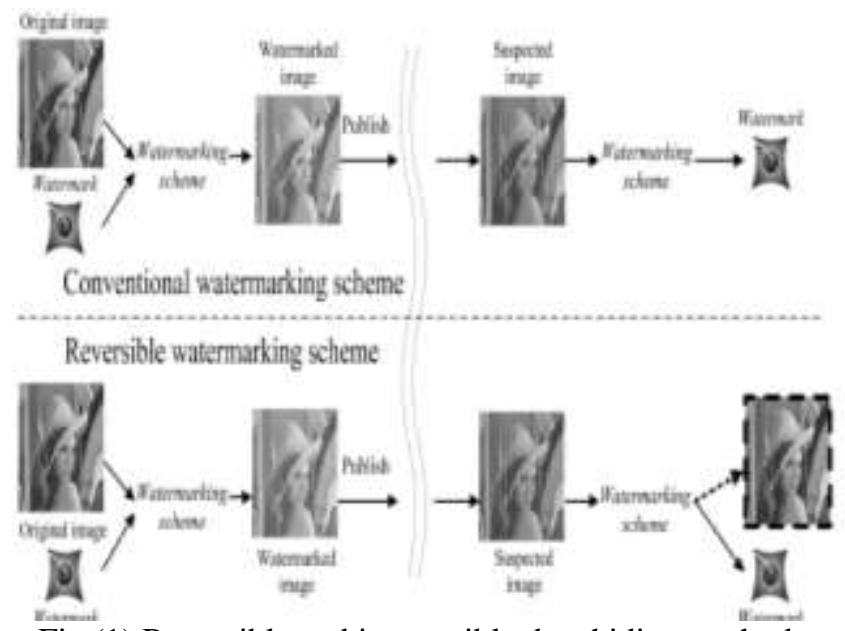

Fig (1) Reversible and irreversible data hiding method 
Data embedding can further classified as irreversible and reversible. In irreversible method loss of information due to embedding process is permanent and original host image can't be retrieved. While in reversible method both embedded image and host image are reconstructed properly. Perhaps both irreversible and reversible embedding techniques try to maintain the perceptual quality of the output image (i.e., embedded with data) at the highest possible level while embedding as many external information as possible into the image. Reversibility is an attractive and beneficial feature particularly for those applications dealing with crucial and sensitive information such as medical images, military images, forensic, and valuable artwork.

Basically data hiding and scrambling both are explored separately. While imperceptibility and output image quality is a matter of interest in the conventional reversible data embedding methods, it is no longer a concern in the joint approach. On the contrary, joint approach should be for data embedding. The main limitation of this approach able to severely degrade the perceptual quality of the image by embedding external information into it while being able to reconstruct the original content. However advancement in digital media usage and availability of high computational tool to manipulate digital data leads to growing interest in using the feature of both disciplines. Integrating both the techniques leads to huge computational requirement to reconstruct the original image and embedded data without losing information. Though it is tough task to build the original image from scrambled image many researchers take advantages of combined features of both the disciplines. These features can be utilized by some of areas such medical and military data transmission.

In conventional method data embedding is done by converting image into new domain or finding some feature of the image to modify. Instead of that in Unified Data embedding and scrambling i.e UES vacate the pixel to insert the external information. First checker board prediction method is used to predict pixel intensity. 25\% pixel from each alternate row and column are stored as reference pixels, by using these $25 \%$ pixel remaining $75 \%$ pixels are predicted in two passes. Then prediction error is calculated to determine whether the predicted pixel is to be vacating for insertion of external data or not. With the help of structure side information original image is reconstructed from scrambled image.

The rest of the paper is structured as follows: Section II have literature review Section III presented the proposed method and the discussion on achieving desirable output image quality. Section IV experimental results are presented. Section V concludes this paper.

\section{LITERATURE REVIEW}

Data hiding are a group of techniques used to put a secure data in a host media (like images) with small deterioration in host and the means to extract the secure data afterwards. For example, Steganography is one such pro-security innovation in which secret data is embedded in a cover image. Reversible data-hiding insert information bits by modifying the host signal, but enable the exact (lossless) restoration of the original host signal after extracting the embedded information. The best policy of ensuring the security of secret information traveling on the Internet is to avoid any attention and suspicion of the hacker. That is to say, the safest method to keep messages transmitted through open channels from leaking out is to encrypt them into a meaningful content and this is where steganography/data hiding comes into play. Reversible data embedding methods are usually proposed in the spatial domain where an image is losslessly stored as an array of raw pixel values, but also occasionally in the compressed domain and frequency domain. The main advantage of spatial domain is its simplicity. Therefore spatial schemes have low computational complexity and consequently need less computational time. The reversible methods in the spatial domain can be further into three sub-categories, namely, compress-and-append, expansion based (EB) and Histogram shifting (HS).

The first generation of reversible data embedding is compress-and-append. The main idea of these methods is to find a losslessly compressible subset A (e.g., LSB bit-plane) in the host content (e.g., image, video and audio file), then replaces A by its compressed representation A, and exploits the vacated space for data embedding. The main limitation of this approach is its limited payload (i.e., the number of embeddable bits), which is generally lower than that of DE and HS. On the other hand, the essence of EB technique is to create some features that are representable by small magnitudes using a decorrelation function [4]. Last but not least, histogram shifting consists of three steps: 1) identify peak and zero points in the pixel value histogram of the host image; 2) vacate space for data insertion by shifting the bins between the zero and peak points, and; 3)embed information by using adjacent bin values [5].

A scalable scrambling method with reversible data hiding functionality was proposed in DCT compressed domain was proposed by wong. Among the available image and video compression standards, the DCT based standards remain the mostly considered ones due to their high quality to compression ratio and the number of existing hardware that support these formats. Therefore, there is a need of continued research in data hiding for the future of DCT based compressed 
contents. Most of the existing scrambling methods focused on security and computation complexity but neglected visual appearance of the scrambled image. For image vendor, it is desirable to produce an image that shows partial detail to attract interested parties while preventing unauthorized, without reconstruction, without the decoding key. Scalable scrambling is a solution to such scenario because it produces image with desired level of distortion while preserving some visual details. At the same time, it will be beneficial to insert information such as resolutions of the image available for purchase, price list, vendor's information, ownership, etc. that stays intact with the partially scrambled image. To the best of knowledge, the scrambling method by Takayamaet al is the only existing work that provides scalable scrambling feature in the DCT compressed domain.

Instead of attempting to suppress quality degradation due to data insertion, Wong utilized this distortion to achieve scalable scrambling effect. In his work, a scalable scrambling method in the DCT compressed domain was proposed while inserting external information into the content, and this method is the first attempt of its kind. It is achieved by scalable scrambling effect by scrambling DC components using a window of varying size while external information is encoded by imposing a specific relationship on the cardinality of two adjacent coefficient blocks. Ong et al. proposed scalable visual quality degradation, by mapping all pixels in the predetermined block to their corresponding mirror values for data embedding purposes Quality of the embedded-scrambled image is further degraded by shuffling pixels in blocks of predefined size

\section{PROPOSED METHOD}

First, an efficient pixel intensity value prediction method, called Checkerboard Based Prediction (CBP), is proposed to accurately predict $75 \%$ of the pixels in the host image prior to data embedding. Then, data embedding is achieved by directly replacing the pixel values by the external information. The proposed method consists of three main processes, namely, pixel value prediction, information embedding, and reconstruction. These processes are elaborated in the following sub-sections.

\section{A. Checkerboard Based Prediction (CBP)}

Pixel intensity value prediction is widely used in image processing. There are numbers of predictor available such as the predictor used in JPEG-LS [6] and MED (Median Edge Detection) which is also known as LOCO-I (LowComplexity Lossless Compression) [7], [8] are the two earliest predictors proposed in 1992 and 1996, respectively. Further some new methods are developed as GAP (Gradient Adjusted Prediction) [9], Graham [10], DARC (Differential Adaptive Run Coding) [11], GBSW (Gradient-Based Selection and Weighting) [12], and GBTA (Gradient-Based Tracking and Adapting) [13].

In the pixel intensity value prediction two parameters are essential and crucial for accurate pixel value estimation. In particular, they are: (a) the distance from the target pixel - since closer neighbouring pixels have higher correlation with the target pixel [refer to Fig. 2(a)], and; (b) the bidirectional information around the target pixel - since by knowing the value of both the previous and next pixels in various directions, the pattern of the target pixel can be accurately approximated [refer to Fig. 2(b)]. Therefore, CBP (Checkerboard Based Prediction) is proposed as an efficient pixel estimation technique which can be independently exploited in various applications, including decorrelation, compression, data embedding, etc.
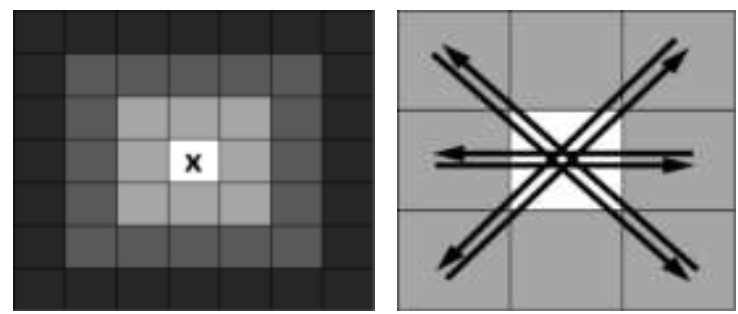

Fig2. Essential parameters for accurate pixel prediction. (a) Distance from the target pixel. (b) Bidirectional information around the target pixel.

First, every other row and column of pixels are stored to be utilized as the reference points to predict the rest of the pixels (i.e., store $25 \%$ of the pixels in raw values to predict the remaining $75 \%$ ) as shown in Fig. 3(a). Then, pixel value estimation is invoked in two passes. In the first pass, pixels marked as ' $\mathrm{X}$ ' in Fig. 3(b) are predicted. Next, those slightly shaded pixels marked as ' $\mathrm{O}$ ' in Fig. 3(c) are predicted in the second pass.

The pixel values are estimated using Eq. (1) and Eq. (2) in the first and second passes, respectively, where rnd(.) denotes the rounding operation. Here, the symbols W, E, N, NW, NE, S, SW, and SE denote the neighbouring pixels at 
west, east, north, North-west, north-east, south, south-west, and south-east of target pixel 'X', respectively, as shown in Fig. 4. Then, the prediction errors are captured as side information and the locations of the predicted pixels are vacated for data embedding purposes
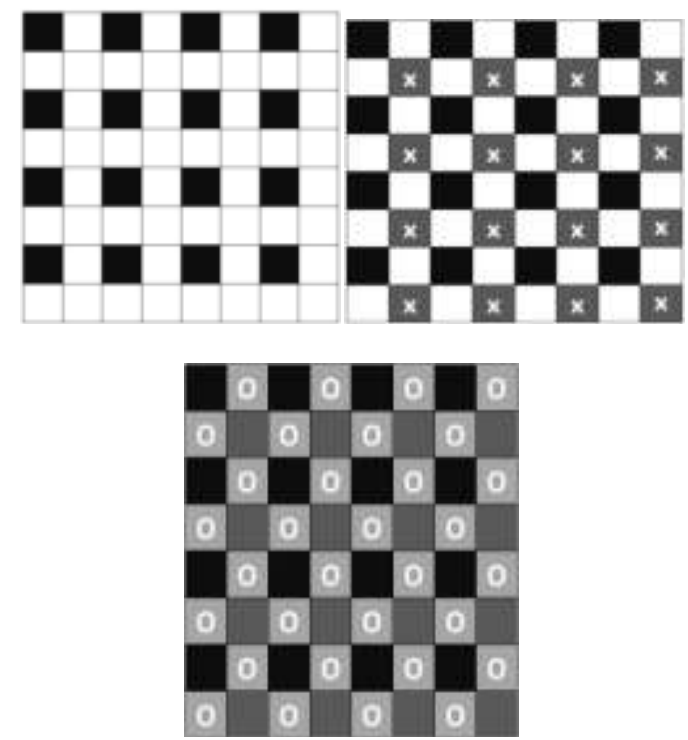

Fig. 3. Scanning order considered in CBP. (a) Stored pixels. (b) First pass of prediction. (c) Second pass of prediction

\begin{tabular}{|c|c|c|}
\hline $\mathrm{NW}$ & $\mathrm{N}$ & $\mathrm{NE}$ \\
\hline $\mathrm{W}$ & $\mathrm{X}$ & $\mathrm{E}$ \\
\hline $\mathrm{SW}$ & $\mathrm{S}$ & $\mathrm{SE}$ \\
\hline
\end{tabular}

Fig. 4. Neighborhood of the target pixel ' $\mathrm{X}$ '

$$
\begin{array}{rlrl}
\mathrm{X}= & \operatorname{rnd}((\mathrm{NW}+\mathrm{SE}) / 2), & \text { if } \mathrm{NE}-\mathrm{SW}>\mathrm{NW}-\mathrm{SE} \\
& \operatorname{Rnd}((\mathrm{NE}+\mathrm{SW}) / 2), & & \text { if..NE- } \mathrm{SW}<\mathrm{NW}-\mathrm{SE} \\
& \mathrm{Rnd}((\mathrm{NW}+\mathrm{NE}+\mathrm{SW}+\mathrm{SE}) / 4), & & \text { otherwise. } \\
\mathrm{X}= & & \\
\operatorname{rnd}((\mathrm{W}+\mathrm{E}) / 2), & \text { if } \mathrm{N}-\mathrm{S}>\mathrm{W}-\mathrm{E} \\
\operatorname{rnd}((\mathrm{N}+\mathrm{S}) / 2), & \text { if } \mathrm{N}-\mathrm{S}<\mathrm{W}-\mathrm{E} \\
\operatorname{rnd}((\mathrm{W}+\mathrm{N}+\mathrm{E}+\mathrm{S}) / 4), & \text { otherwise }
\end{array}
$$
$\mathrm{X}=\operatorname{rnd}((\mathrm{W}+\mathrm{E}) / 2), \quad$ if $\mathrm{N}-\mathrm{S}>\mathrm{W}-\mathrm{E}$

B. Unified Embedding-Scrambling (UES):

The process flow of the proposed embedding-scrambling method is summarized in Fig. 5. First, the proposed CBP is utilized to predict pixel values in the image. Next, each prediction error, denoted as ep for the rest of the presentation, is computed as $e p=x-x p$ where $x$ and $x p$ are the original and predicted value by CBP, respectively.

Then, ep is analysed to decide if the corresponding pixel locations suitable for data embedding. In particular, if ep falls within a pre-defined range as expressed in Eq. (3)

$$
-\varepsilon \leq \mathrm{ep} \leq \varepsilon
$$

In particular, we classify all pixels in an image into three categories, namely, (a) not-predicted (NP), (b) predicted but not embedded (PN) and, predicted-and-embedded (PE). Here, the set of NP consists of all the reference points in every other column and row, which are utilized to predict the rest of the pixels using the proposed CBP method. Next, PN refers to a pixel whose ep fails the condition in Eq. (3). In other words, PN is a pixel that cannot be predicted accurately by the proposed CBP method, and it is not considered for data embedding. Thus, PN holds the original pixel value. Finally, PE refers to a pixel that satisfies Eq. (3), and it is utilized for data embedding. Here, the prediction errors ep are stored as side information to reconstruct the original image. 


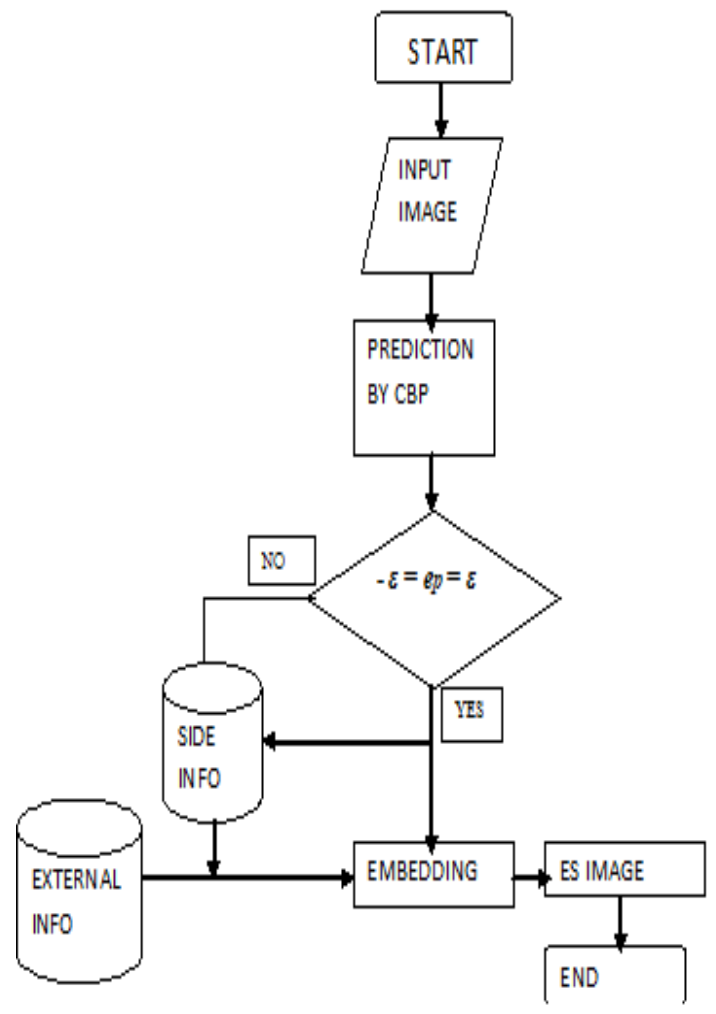

Fig 5 Process flow of proposed method

\section{Extraction and Reconstruction}

Side information is required to extract the embedded information and to reconstruct the original host image. The most important role of our side information is to tell apart if a pixel is the embedded information or it contains the actual pixel intensity value. In the case where the actual intensity value is stored (i.e., NP and PN), no information is embedded. In other words, the pixel value is either not predicted (NP) or it is predicted but not vacated for data embedding(PN) because the error fails Eq.(3).Therefore, there is no embedded information to be extracted and there is no modification on the host image which needs to be restored.

\section{IV.EXPERIMENTAL RESULTS}

As a proof of concept, the proposed method is implemented using Matlab Version 7.14.0.739 on a Intel Core i3 2.00 MhZ with 4GB of memory. The benchmark image datasets, namely: 7 images (each of dimension $512 \times 512$ pixels) from the USC-SIPI standard test image dataset is considered for experimental purposes.
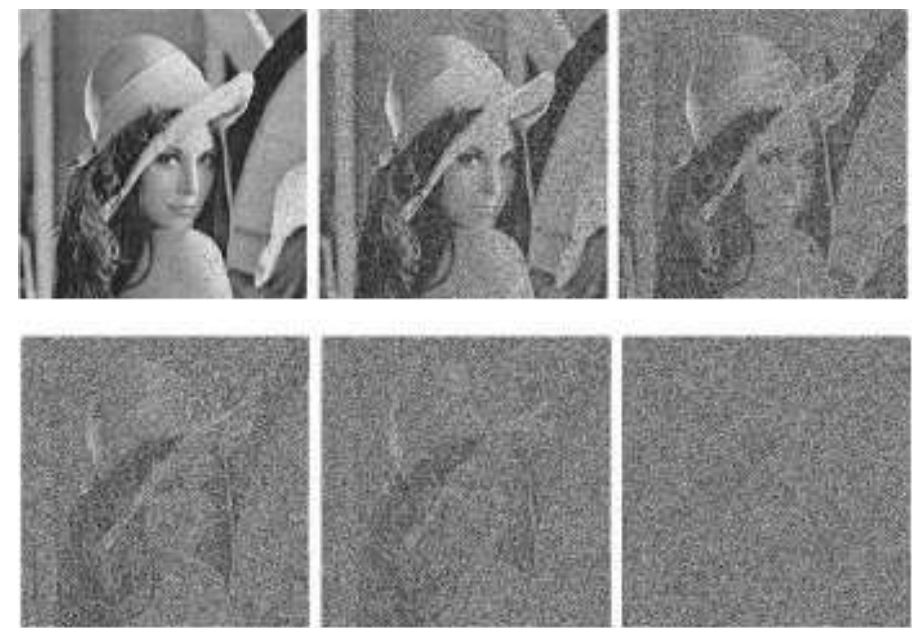

Fig. 5(a) Embedded-scrambled image using UES 

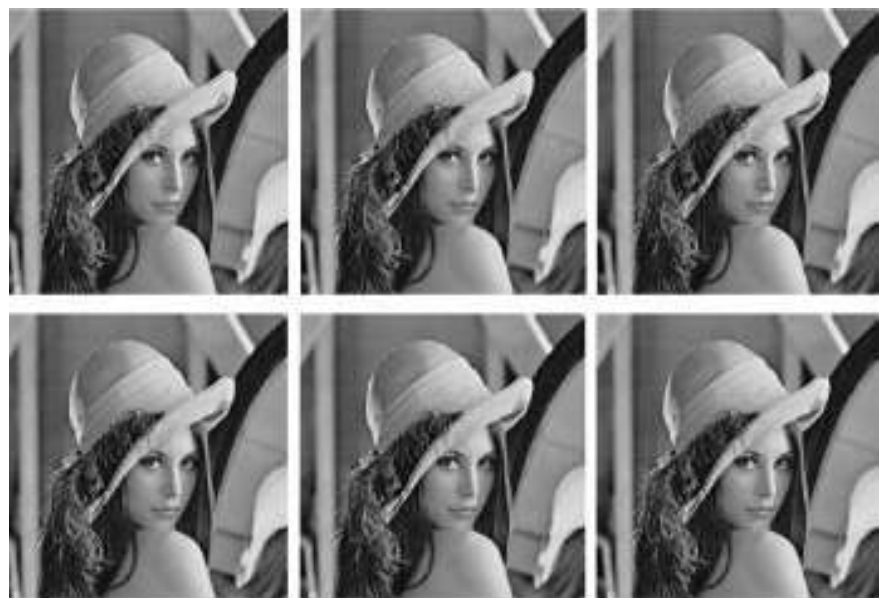

Fig.5(b) Reconstructed image using UES method

It is verified by visual inspection that our method is able to severely degrade quality of the host image by embedding external information into it and the distortion level can be controlled by changing $\varepsilon$ as well as L (i.e., level of processing). It is also verified that the embedded information can be completely extracted, and quality of the reconstructed image is controllabled. When $\varepsilon=0$ and $\mathrm{L}=1$, the general appearance of the original image is still perceivable as suggested by Fig. 6 . By increasing the value of $\varepsilon$ and $\mathrm{L}$, more distortion is introduced to host image as more pixels are exploited for data embedding. Hence the perceptual quality of the embedded-scrambled image decreases. In particular, when $\varepsilon$ increases, Eq. (3) becomes less restrictive, hence more pixels become PE. On the other hand, when L increases, more pixels (in the sub-images) are considered and tested against Eq. (3), hence possibly increasing the number of PE's. Nevertheless, the change in $\varepsilon$ has greater impact on quality of the embedded-scrambled image when compared to $\mathrm{L}$ due to the sharp distribution of the prediction errors.

\section{CONCLUSION}

The advance method of reversible data embedding i.e unified data embedding and scrambling USE is proposed. The proposed method gets higher payload while degrading the perceptual quality of the host image to the desire level. First the pixel predictor was proposed which can accurately predict $75 \%$ pixel value with reference to $25 \%$ pixel. The proposed pixel prediction method achieved accurate prediction, up to twice the accuracy of the conventional methods considered. The embedding and scrambling of data was done by selecting the pixel with reference to prediction error and these pixels were vacate for data embedding, while their prediction errors were stored as side information. The embedded is image was reconstructed using side information. Experimental results confirmed that the proposed method is able to completely degrade the perceptual quality of the host image by embedding external information into it. Experimental results also indicated that the proposed method is able to recover the host image after imposing severe degradation by embedding huge amount of external information.

\section{REFERENCES}

[1] Chu YH, Chang S. Dynamical cryptography based on synchronized chaotic systems. IEE Electronics Letters 1999;35(12):974-5.

[2] Highland HJ. Data encryption: a non-mathematical approach. Computers and Security 1997;16(5):369-86.

[3] I. Cox, M. Miller, J. Bloom, J. Fridrich, and T. Kalker, Digital Watermarking and Steganography. San Mateo, CA, USA: Morgan Kaufmann, 2008.

[4] W. Hong, T. Sh. Chen, and Ch. W. Shiu, "Reversible data hiding for high quality images using modification of prediction errors," J. Syst. Softw., vol. 82, no. 11, pp. 1833-1842, 2009.

[5] Z. Ni, Y. Q. Shi, N. Ansari, and W. Su, "Reversible data hiding,” IEEE Trans. Circuits Syst. Video Technol., vol. 16, no. 3, pp. 354-362, Mar. 2006.

[6] G. K. Wallace, "The JPEG still picture compression standard,” IEEE Trans. Consumer Electron., vol. 38, no. 1, pp. 1988-1994, Feb. 1992.

[7] M. J. Weinberger, G. Seroussi, and G. Sapiro, "LOCO-I: A low complexity, context-based, lossless image compression algorithm," in Proc. DCC, 1996, pp. 140-149.

[8] M. J. Weinberger, G. Seroussi, and G. Sapiro, "The LOCO-I lossless image compression algorithm: Principles and standardization into JPEGLS," IEEE Trans. Image Process., vol. 9, no. 8, pp. 253-266, Aug. 2000.

[9] X. Wu and N. Memon, "Context-based, adaptive, lossless image coding," IEEE Trans. Commun., vol. 45, no. 4, pp. 437-444, Apr. 1997.

[10] R. E. Graham, "Predictive quantizing of television signals," IREWescon Conv. Rec., vol. 1, pp. 147-157, Jan. 1958.

[11] B. Gandhi, C. Honsinger, M. Rabbani, and C. Smith, "A proposal submitted in response to call for contributions for JTC," ISO Working Doc. ISO/IEC JTC1/SC29/WG1 N204, 1995.

[12] J. Knezovic and M. Kovac, "Gradient based selective weighting of neighboring pixels for predictive lossless image coding," in Proc. 25th Int. Conf. Inf. Technol. Inter., Jun. 2003, pp. 483-488.

[13] R. M. Rad, A. Attar, and A. Shahbahrami, “A predictive algorithm for multimedia data compression,” Multimedia Syst., vol. 19, no. 2, pp. 103115, 2013. 